\title{
Usefulness of intraoperative cortical blood flow measurement by heat clearance method for monitoring cerebral ischaemia during therapeutic carotid ligation
}

\author{
T HAYAKAWA, ${ }^{*} \mathrm{~K}$ YAMADA,${ }^{*}$ Y IWATA,$\dagger$ A KATO,${ }^{*}$ T YOSHIMINE, ${ }^{*}$ Y USHIO,${ }^{*}$ \\ S NAKATANI, ${ }^{*}$ T IKEDA, ${ }^{*}$ H MOGAMI* \\ From Department of Neurosurgery, Usaka University Medical School* and Department of Neurosurgery, \\ Minoh City Hospital, $\dagger$ Osaka, Japan
}

\begin{abstract}
SUMmARY Changes of regional cortical blood flow (rCBF) was monitored by the heat clearance method during the operation of cervical carotid ligation combined with superficial temporal artery and middle cerebral artery anastomosis for the treatment of inaccessible aneurysms of the internal carotid artery in eight patients. There were three types of rCBF responses upon closure of the internal carotid artery, with or without bypass blood flow. Based on the responses, the internal carotid artery was ligated abruptly or occluded gradually. The usefulness of the method for monitoring cerebral ischaemia for therapeutic carotid ligation is discussed.
\end{abstract}

Cervical carotid ligation is effective in the treatment of intracavernous and/or giant aneurysm of the internal carotid artery inaccessible to direct surgery.' However, the immediate or delayed occurrence of cerebral ischaemic complications is a serious problem..$^{2-4}$ To avoid complications due to low perfusion, it is important to assess the collateral circulation after carotid ligation before and during operation. Many methods of assessment have been reported, but each has disadvantages and their reliability has not been established.

Recently, we found that measurement during the operation of regional cortical blood flow ( $\mathrm{rCBF}$ ) by the heat clearance method ${ }^{5-7}$ at craniotomy for combined extracranial and intracranial bypass surgery is useful for this purpose.

\section{Material and methods}

Regional CBF was monitored in eight patients who had giant or intracavernous aneurysms of the internal carotid

Address for reprint requests: Toru Hayakawa, MD, Department of Neurosurgery, Osaka University Medical School, 1-1-50 Fukushima, Fukushima-ku, Osaka 553, Japan

Received 12 July 1984 and in final revised form 16 November 1984.

Accepted 23 November 1984 artery undergoing treatment by cervical carotid ligation combined with superficial temporal artery and middle cerebral artery anastomosis. Before operation, Matas test and contralateral carotid angiography were performed to assess spontaneous collateral blood supply after carotid ligation. In the Matas test, the EEG was recorded during percutaneous compression of the carotid artery for more than 15 minutes; and changes of neurological signs and EEG were studied. Cross-filling of the cerebral arteries during the compression of the carotid artery on the side of the aneurysm was studied by angiography after an injection of contrast medium into the opposite carotid artery. Operative procedure: Under general anaesthesia, the bifurcation of the carotid artery in the neck was exposed and superficial temporal artery to middle cerebral artery anastomosis was performed through the ipsilateral frontotemporal craniotomy. One or two thermal diffusion flow probes (Plate-type, outside diameter $13 \mathrm{~mm}$, UniqueMedical Co. Ltd., Komae, Tokyo, Japan) were placed on the surface of the frontal and/or temporal lobes exposed by craniotomy for superficial temporal artery to middle cerebral artery anastomosis. Attention was paid to avoid contact between large surface vessels and the probes. The probes were then covered with wet cotton pledgets, taking care not to deform the cortex. The output direct current from the thermocouple probe was amplified by an amplifier (UM meter: UM 2000, Unique-Medical Co. Ltd.) and recorded by XY recorder (Watanabe Multicorder MC 6601, Watanabe Instruments Corp.). The heating current for the thermocouple heating circuit was $20 \mathrm{~mA}$ and the sensitivity was adjusted as $10 \mu \mathrm{V}$ of probe output 
was equivalent to $1.0 \mathrm{~cm}$ or $3.0 \mathrm{~cm}$ on a recording graph. Systemic arterial pressure was monitored with a catheter inserted into the radial artery and cervical internal carotid artery pressure was monitored with a 21-gauge needle inserted into the distal part of the exposed internal carotid artery in the neck.

After the anastomosed superficial temporal artery had been temporarily occluded by a clip, the internal carotid artery was clamped just distal to the bifurcation of the common carotid artery, and the changes of rCBF and the arterial pressures were recorded. A few minutes after, the clip on the anastomosed superficial temporal artery was removed, and a further few minutes later, the clamp on the internal carotid artery was released. These procedures were repeated twice.

Based on the data obtained, ligation of the internal carotid artery was performed abruptly, or a Selverstone clamp was placed on the internal carotid artery for later gradual occlusion of the internal carotid artery.

The results of surgery were evaluated by computed tomography and cerebral angiography, performed more than two weeks after surgery.

\section{Results}

There were three types of $\mathrm{rCBF}$ responses to internal carotid artery occlusion. Type A: While the anastomosed superficial temporal artery was occluded, no change of $\mathrm{rCBF}$ was observed upon closure of the cervical internal carotid artery (fig 1, two cases); Type B: Decrease of rCBF by closure of internal carotid artery was cancelled by opening the anastomosed superficial temporal artery (fig 2, three cases), and Type (: Decrease of rCBF by closure of internal carotid artery was not compensated by opening the anastomosed superficial temporal artery (fig 3, three cases). Systemic arterial pressure was not usually changed throughout the procedures. The distal internal carotid artery pressure (back pressure) was decreased to $11 \%$ to $47 \%$ of the preclamped level in the seven cases in which it was measured.

In the cases of Type A, the internal carotid artery was ligated abruptly: in those of Type C, it was occluded gradually with a Selverstone clamp in the post-operative period. Referring to the findings of pre-operative EEG and Matas test, angiographical cross filling and intra-operative internal carotid artery back pressure, the internal carotid artery was ligated abruptly in two cases of Type B, and in one case, the internal carotid artery was occluded gradually. In the former two cases; ligation was abrupt because the Matas test was negative, angiographical cross filling was good, reduction of distal intravascular pressure of the carotid artery during temporary carotid occlusion was less than $30 \%$ from control, and the results indirectly suggested sufficient development of spontaneous collaterals after carotid occlusion. The table summarises the results of the cases.

There were no complications due to low perfusion after operation, but an embolism occurred in one case. In this case, a 31-year-old woman with an intracavernous carotid aneurysm, superficial temporal artery to middle cerebral artery anastomosis was performed with application of a Selverstone clamp to the right internal carotid artery. The clamp was advanced to about $80 \%$ occlusion of the internal carotid artery on the 2 nd postoperative day. The patient tolerated this procedure well and no

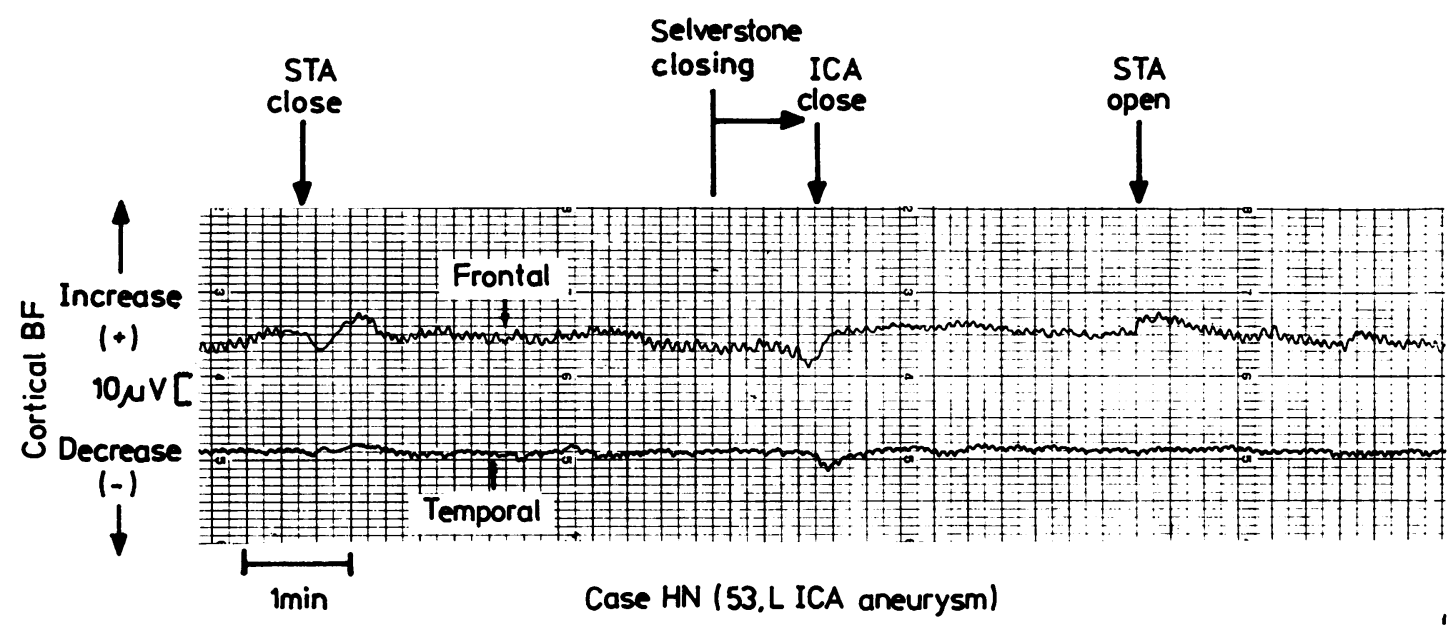

Fig 1 Recoraing of the Type A response. Regional cortical blood flow shows almost no change by occlusion of the internal carotid artery and anastomosed superficial temporal artery. 

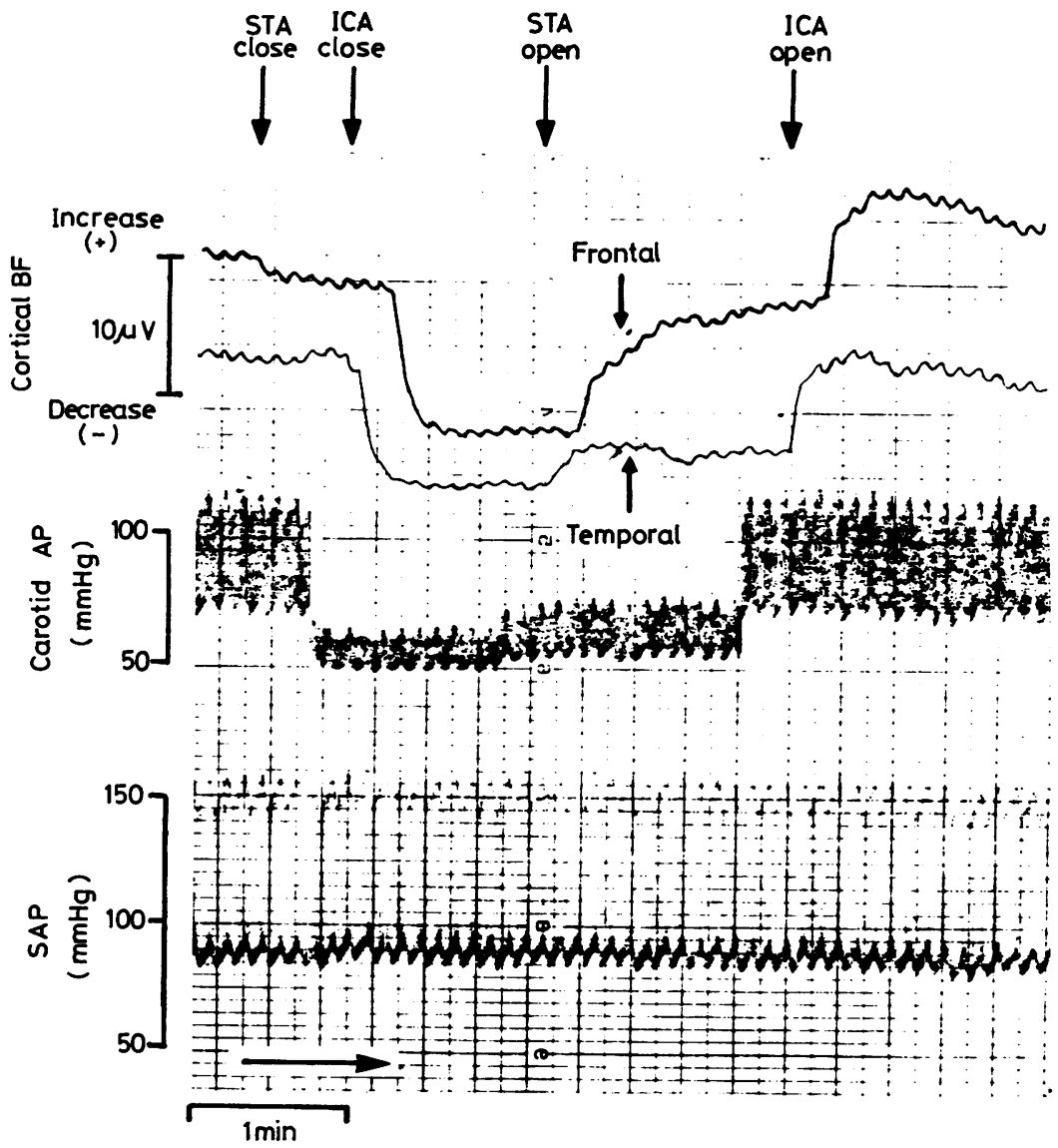

Fig 2 Recording of the Type B response. Regional cortical blood flow (rCBF) and internal carotid artery stump pressure decrease by closure of internal carotid artery, but the decrease of $r C B F$ recovers by opening the anastomosed superficial temporal artery, particularly in the frontal region.

neurological changes ensued. However, early the next morning, left hemiparesis and dysarthria occurred and angiography showed embolic stenosis in the stem of the right middle cerebral artery. After physical therapy, she was discharged with mild weakness of the left upper extremity. ${ }^{15}$

Disappearance of rapid and marked enhancement with contrast medium in the aneurysmal cavity on computed tomography, and failure to demonstrate the aneurysm on cerebral angiography after surgery suggested that the aneurysms were thrombosed.' Development of collateral flow through surgical bypass was demonstrated on cerebral angiograms in all cases where the examination was performed.

\section{Discussion}

In the Cooperative Study of Intracranial Aneurysms and Subarachnoid Hemorrhage, ${ }^{3}$ the incidence of ischaemic neurological complications after carotid occlusion was $233 / 814(28.6 \%)$; the rate was higher in older patients than in younger patients, higher with abrupt ligation ( $34 \%$ ) than with gradual occlusion $(25 \%)$, and significantly higher with internal carotid occlusion (49\%) than with common carotid occlusion (28\%). Recently, Heros et al ${ }^{84}$ indicated that the rates of ischaemic complications of cervical carotid ligation reported in the literature ranged from $7 \%$ to about $50 \%$, with an average about $25 \%$, and, of these, roughly half were serious and permanent. Two factors have been considered as the main causes of cerebral ischaemic complications; one is low perfusion and the other is thrombo-embolism. ${ }^{3}$

To avoid ischaemic complication due to low perfusion, the technique of gradual occlusion of the carotid artery and/or the combined surgery of 


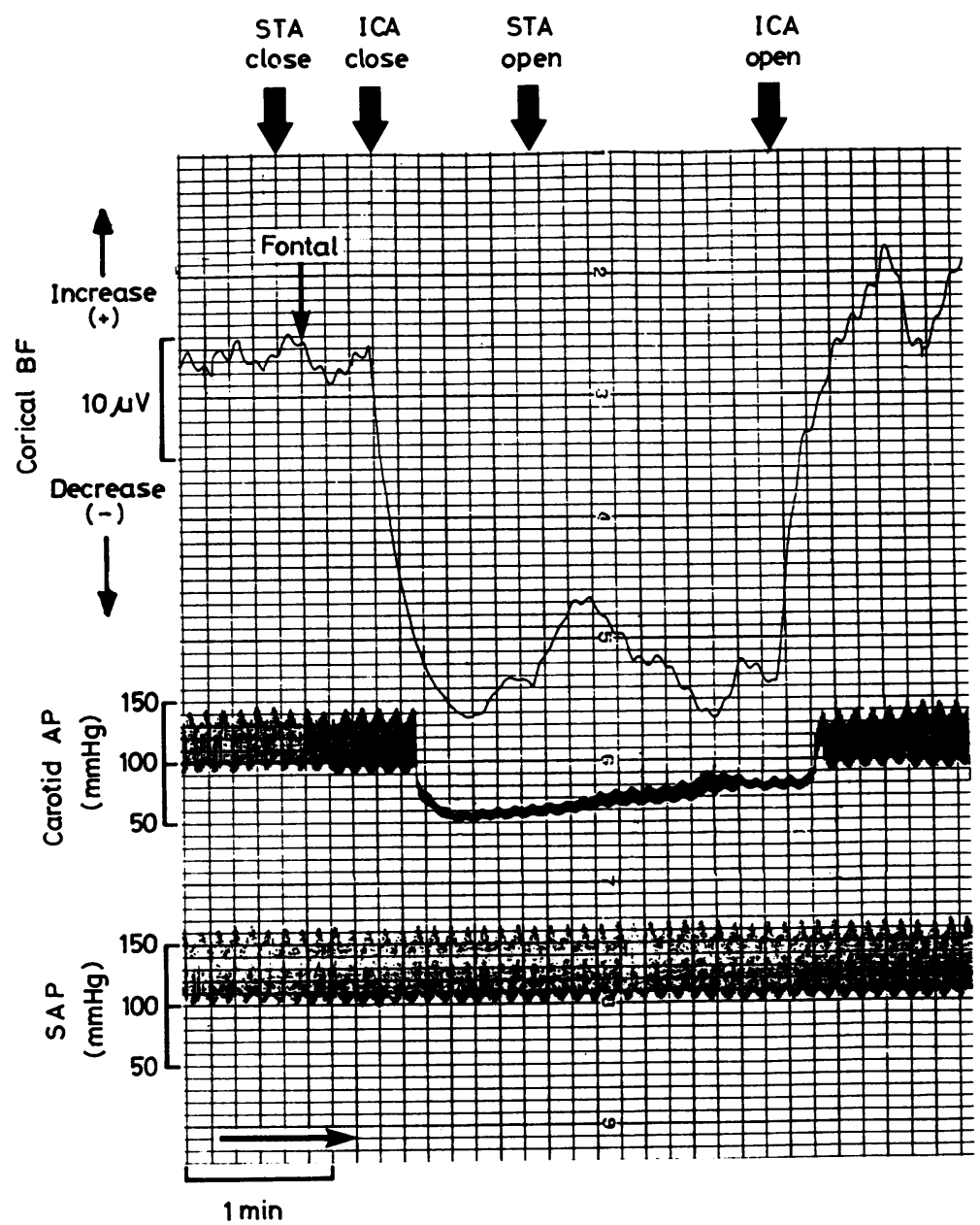

Fig 3 Recording of the Type C response. The decreases of $\mathrm{CBF}$ and internal carotid artery stump pressure by closure of internal carotid artery do not recover by opening of the anastomosed superficial temporal artery.

extracranial and intracranial bypass have been employed. The effectiveness of gradual occlusion of the carotid artery to aid the development of a collateral circulation and to prevent acute ischaemic complication was demonstrated in the cooperative study and by others. ${ }^{310}$ Many kinds of instrument, for example the Selverstone clamp, or the Crutchfield clamp, have been developed for this purpose. Extracranial and intracranial bypass surgery, especially superficial temporal artery and middle cerebral artery anastomosis, is a relatively simple and safe procedure, and can contribute a significant blood flow to the ischaemic cerebral hemisphere. It is logical to believe that this procedure is effective in preventing acute ischaemia in patients with marginal collateral circulation. Furthermore, extracranial and intracranial bypass may be even more effective in preventing late ischaemic complication after carotid ligation,${ }^{4}$ for the bypass blood flow would increase later in the follow-up period, in response to the demands of the brain." "Based on the view, extracranial and intracranial bypass surgery has been usually combined before carotid ligation following the development of microsurgical techniques in recent years. ${ }^{1213}$

However, a few reports have appeared recently in which ischaemic complication occurred occasionally in cases of gradual carotid occlusion combined with superficial temporal artery and middle cerebral 
Table Summary of the cases

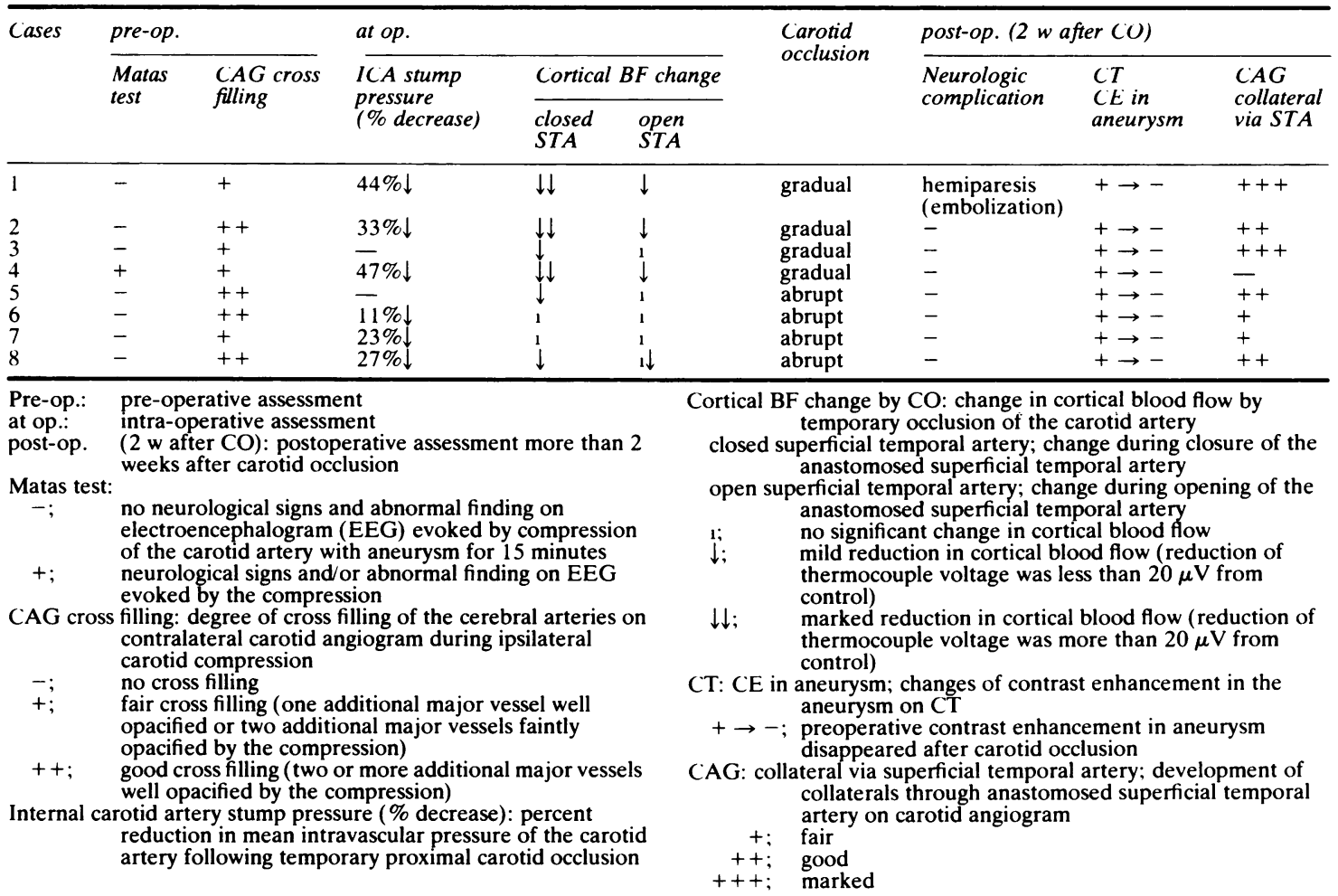

artery anastomosis; thromboembolism was considered to be the cause of such cerebral ischaemia. ${ }^{8414}$ As mentioned above, we also encountered a cerebral thrombo-embolic complication in a patient with an intracavernous carotid aneurysm who was treated by gradual occlusion of the internal carotid artery combined with superficial temporal artery and middle cerebral artery anastomosis. ${ }^{15}$ Based on available evidence, we have suggested that in the presence of a sufficient collateral blood supply after combined extracranial and intracranial bypass surgery, abrupt internal carotid artery ligation may reduce the risk of thrombo-embolic complications.

As to low cerebral perfusion caused by carotid ligation, Miller et al indicated that about $80 \%$ patients will tolerate acute occlusion of one internal carotid artery but $20 \%$ will not. ${ }^{16}$ In order to ligate the carotid artery safely, it is necessary to assess the collateral circulation after carotid ligation before and during operation. For this purpose, there exist numerous examinations and tests: EEG-Matas test, ${ }^{1016}$ angiography for cross filling, ${ }^{17}$ ophthalmodynamometry, ${ }^{10}$ ophthalmoplethysmography, ${ }^{18}$ intravascular balloon-Matas test, ${ }^{14}$ Doppler flowmetry with compression test, ${ }^{20}$ regional cerebral blood flow study with radioisotope, ${ }^{1617}$ electromagnetic flow measurement, ${ }^{21}$ back pressure measurement of the internal carotid artery, ${ }^{10}{ }^{17}$ etc. Though every method for the assessment has some advantages, it also has some defects and problems. In recent years when extracranial and intracranial bypass surgery has become routinely combined with this treatment, the development of collateral circulation after carotid ligation and anastomosis needs to be evaluated after bypass surgery. A new reliable method for this assessment is required.

Regional blood flow measurement with thermal diffusion flow probe (heat clearance method) was originally developed by Gibbs in 1933..22 Many have improved the method, and the modern technique using thermocouples has been widely applied, mainly in the experimental studies. The advantages of the method for regional blood flow measurement are (1) it is a simple and easy technique using small apparatus, (2) the ability to measure regional blood flow dynamically and continuously, (3) the method is non-invasive when a plate-type probe is used, (4) it is not necessary to use radioisotope. These advantages indicate that this method is suitable for clinical intra-operative measurement of the regional blood 
flow of tissues. However, there are few reports of clinical use of this method for cerebral circulation, ${ }^{23}{ }^{24}$ except in experimental conditions. There has been no report of practical application of this method except for recent studies by Carter et al..$^{25} 26$ They applied this technique using a Peltier stack in the neurosurgical field and indicated that intraoperative evaluation of $\mathrm{rCBF}$ with this method may be of value in estimating the safety of temporary occlusion of the major cerebral vessel for bypass or aneurysm surgery. In the present paper, we demonstrated the practical usefulness of the rCBF measurement by the heat clearance method for monitoring the development of collateral blood supply after carotid ligation.

The disadvantage of the method is inaccuracy in quantitative measurement and difficulty in expressing regional blood flow data as absolute values (such as $\mathrm{ml} / 100 \mathrm{~g} / \mathrm{min}$ ). However, this method is useful enough to check whether a decrease of $\mathrm{rCBF}$ during carotid ligation occurs or not, and whether it may be compensated by opening bypass flow. Another weak point of this method is that the measurement is limited to the exposed cortical surface during bypass surgery. Furthermore, ischaemia in the deeper regions cannot be detected by cortical blood flow measurement with plate-type flow probes. However, the ischaemia caused by internal carotid artery occlusion occurs mainly in the middle cerebral artery territory where craniotomy is performed for superficial temporal artery and middle cerebral artery anastomosis, and low perfusion caused by internal carotid artery occlusion usually takes place diffusely, though thrombo-embolic ischaemia may occur locally in a small region, that is perforator vessel infarction. It is concluded that rCBF measurement by the heat clearance method at craniotomy for combined superficial temporal artery and middle cerebral artery anastomosis offers sufficient information regarding ischaemia caused by internal carotid artery ligation.

Grateful acknowledgement is made to members in Departments of Neurosurgery, Osaka University Hospital and Kwansai-Rosai Hospital for collaboration in this study. This investigation was supported, in part, by a grant in aid for Fundamental Scientific Research from the Education Ministry, Japan.

\section{References}

' Kataoka K, Yamada K, Nakao K, et al. Digital subtraction angiography and dynamic computed tomography for evaluating the hemodynamics in cases of giant intracranial aneurysm. Surg Neurol 1983;20:355-60.
${ }^{2}$ Voris HC. Complication of ligation of the internal carotid artery. J Neurosurg 1951;8:119-31.

${ }^{3}$ Nishioka H. Report of the cooperative study of intracranial aneurysms and subarachnoid hemorrhage. Section III, Part 1.J Neurosurg 1966;25:660-82.

${ }^{4}$ Roski RA, Spetzler RF, Nulsen FE. Late complications of carotid ligation in the treatment of intracranial aneurysms. J Neurosurg 1981;54:583-7.

${ }^{5}$ Betz E, Ingvar D, Cassen N, Schmal F. Regional blood flow in the cerebral cortex, measured simultaneously by heat and inert gas clearance. Acta Physiol Scand 1966;67: 1-9.

- Yamaura A. Regional blood flow of cerebral cortex in cat. J Jpn Coll Angiol 1970;10:61-8.

${ }^{7}$ Hayakawa T. Intraoperative measurement of cortical blood flow by heat clearance method. In: Kikuchi $\mathrm{H}$, ed. Neurosurgeons. Vol 4. Tokyo: The Japanese Congress of Neurosurgical Surgeons 1984:183-90.

* Heros RC, Nelson PB, Ojemann RG, Crawell RM, DeBrun G. Large and giant paraclinoid aneurysms; surgical techniques, complications, and results. Neurosurgery 1983;12: 153-63.

${ }^{4}$ Heros RC. Thromboembolic complications after combined internal carotid ligation and extra- to intracranial bypass. Surg Neurol 1984;21:75-9.

${ }^{10}$ Tindall GT, Fleischer AS. Management of aneurysms of anterior circulation by carotid artery occlusion. In: Youmans JR, ed. Neurological Surgery. Philadelphia: Saunders 1982:1697-714.

" Iwata Y, Hayakawa T, Shimizu K, Mukawa J, Mogami $H$, Nukada T, Kimura K, Yoneda S, Takano T, Mitomo M. STA/MCA anastomosis and collateral circulation. Neurol Med Chir 1980;20:701-11.

${ }^{12}$ Gelber BR, Sundt TM Jr. Treatment of intracavernous and giant carotid aneurysms by combined internal carotid ligation and extra- to intra-cranial bypass. $J$ Neurosurg 1980;52:1-10.

${ }_{13}$ Spetzler RF, Schuster H, Roski RA. Elective extracranial-intracranial arterial bypass in the treatment of inoperable giant aneurysms of the internal carotid artery. $J$ Neurosurg 1980;53:22-7.

14 Diaz FG, Ausman JI, Pearce JE. Ischemic complications after combined internal carotid artery occlusion and extracranial-intracranial anastomosis. Neurosurgery 1982;10:563-9.

is Hayakawa T, Iwata Y, Yamada K, et al. Increased risk of cerebral thrombo-embolic complication during gradual carotid artery occlusion combined with extraand intra-cranial bypass surgery in the treatment of an inaccessible carotid aneurysm. Neurol Med Chir 1985;25:23-26.

${ }^{16}$ Miller JD, Jawad K, Jennett B. Safety of carotid ligation and its role in the management of intracranial aneurysms. J Neurol Neurosurg Psychiatry 1977;40:64-72.

17 Jawad K, Miller JD, Wyper DJ, Rowan JO. Measurement of CBF and carotid artery pressure compared with cerebral angiography in asessing collateral blood supply after carotid ligation. $J$ Neurosurg 1977; 46: 185-96.

${ }^{18}$ Schuler JJ, Flanagan P, DeBord JR, Ryan TJ, Castronuovo JJ, Lim LT. The treatment of cerebral ischemia by external carotid artery revascularization. 
Arch Surg 1983;118:567-72.

${ }^{14}$ Hacke W, Zeumer H, Ringelstein EB. EEG controlled occlusion of the internal carotid artery during angiography. Neuroradiology 1981;22:19-22.

211 Yoneda S, Nukada T, Kimura K, et al. Evaluation of cross-circulation through circle of Willis using an ultrasonic Doppler technique. Part 1. Comparison between blood flow velocity by ultrasonic Doppler Flowmetry and angiogram. Stroke 1981;12:478-88.

21 Tindall GT, Odon GL, Cupp HB Jr, Dillon ML. Studies on carotid artery flow and pressure observation in 18 patients during graded occlusion of proximal carotid artery. J Neurosurg 1962;19:917-23.

${ }^{22}$ Gibbs FA. A thermoelectric blood flow recorder in the form of a needle. Proc Soc Exp Biol 1933;31:141-6.

${ }^{23}$ Wüllenweber $\mathbf{R}$. Observations concerning autoregulation of cerebral blood flow in man. Acta Neurol Scand 1965;4 suppl 14:111-5.

${ }^{24}$ Capon A, Martin DH, Noterman J. Évaluation du débit sanguin local dans les tumeurs cérébrales à l'aide d'une méthode de thermodiffusion. Neurochirurgie 1969; 15:569-74.

${ }^{25}$ Carter LP, White W, Atkinson J. Regional cortical blood flow at craniotomy. Neurosurgery 1978;2:223-9.

${ }^{26}$ Carter LP, Erspamer R. Regional cortical blood flow during cerebrovascular surgery. In: Peerless SJ, McCormick CW, eds. Microsurgery for Cerebral Ischemia. New York: Springer-Verlag 1980:40-5. 